\title{
Carnets
}

Revue électronique d'études françaises de l'APEF

Première Série - 5 | 2013

Métamorphoses littéraires

\section{Les métamorphoses de Joseph}

\section{Laurent Pietra}

\section{(2) OpenEdition}

Journals

Édition électronique

URL : http://journals.openedition.org/carnets/8115

DOI : 10.4000/carnets.8115

ISSN : 1646-7698

Éditeur

APEF

Édition imprimée

Date de publication : 1 mai 2013

Pagination : 9-26

Référence électronique

Laurent Pietra, «Les métamorphoses de Joseph », Carnets [En ligne], Première Série - 5 | 2013, mis en ligne le 23 juin 2018, consulté le 01 mai 2019. URL : http://journals.openedition.org/carnets/8115 : DOI : 10.4000/carnets.8115

Ce document a été généré automatiquement le 1 mai 2019.

\section{(c) (i) (8)}

Carnets est mis à disposition selon les termes de la licence Creative Commons - Atribution - Pas d'utilisation commerciale 4.0 International. 


\title{
Les métamorphoses de Joseph
}

\author{
Laurent Pietra
}

1 Joseph est un nom commun, et lorsqu'on se rapporte au personnage biblique, c'est souvent le père de Jésus qui se présente au lieu du premier détenteur du nom, qui est le fils préféré du patriarche Jacob. Un Joseph s'efface toujours derrière un autre Joseph auquel il se substitue (le nom yossef signifie l'ajout et l'effacement; en Genèse 30 23-24, Rachel dit: "Dieu a effacé/asaf ma honte" et "Dieu veuille me donner encore un second fils"). Bien que Joseph ne soit pas le personnage biblique le plus connu, très peu de personnages bibliques eurent une postérité universelle comparable à la sienne: le personnage et son récit (Genèse 37-50) semblent attirer les identifications, nourrir d'autres récits, se métamorphoser constamment et traverser le temps. Paradoxalement, c'est la multiplicité des métamorphoses de Joseph qui fait sa singularité: la singularité narrative se fait singularité historique.

2 Si Joseph a bénéficié d'une élection théologique, philosophique, littéraire et politique, c'est qu'il ne désigne pas un seul individu, une seule chose; c'est un joker qui nomme une pluralité de choses, d'êtres, de situations, de processus. Plus qu'un nom, Joseph est un principe; son étymologie en fait un principe d'augmentation, d'autorité, de métamorphose. C'est donc une foule de personnages, de récits, d'individus, de phénomènes, de concepts qu'on pourra décrire comme joséphiques. Joseph est comme un mythe vécu qui modèle la vie de personnages pour lesquels la limite entre fiction et réalité s'estompe, ainsi que celle entre exclusion et élection, sans impliquer pour autant oracle ou destin. Des auteurs se rapportent à Joseph tantôt pour incarner tel ou tel concept, telle situation ou tel processus historiques, tantôt pour s'identifier à lui, pour agir comme lui.

Cette figure salvatrice qui permet d'échapper au mal fournit un conseil et rend pensable une nouvelle institution pour les rescapés, une métamorphose du mal en bien, dont le résultat est cependant toujours incertain. Les métamorphoses du Livre biblique en nouveaux récits renvoient alors à des situations historiques critiques et ne se laissent pas réduire à des suites romanesques. L'histoire de Joseph ne donne pas seulement à penser, elle explique des comportements, des institutions, donnant à l'interprétation des textes et des expériences précédents le sens d'une survie, d'une échappée possible hors des 
maux d'une époque, à l'opposé d'une lecture oraculaire et destinale. La valeur herméneutique, philosophique et politique, de cette longue "tradition" joséphique permet ainsi de lire et de mieux comprendre certaines réalités, jusqu'aux plus contemporaines.

Le récit joséphique est une matrice et comme la plupart des textes bibliques un texte de textes; dans le récit lui-même, la forme de la matrice est celle de l'Egypte, du Nil et de son delta; le tissu des textes trouve une image dans la tunique bariolée de Joseph; vêtement d'une beauté incomparable pour le jeune homme extraordinairement beau qui le tient de sa mère Rachel, elle aussi extraordinairement belle (le hen biblique), morte à la naissance du dernier frère Benjamin. Cette tunique bariolée symbolise l'exclusion comme l'élection, la singularité et la multiplicité qui engendrent l'universalité de ce récit. Cette histoire vient en effet de peuples et de textes plus anciens que lui, comme le Conte des deux frères (Lefebvre, 1988); plusieurs éléments du récit joséphique englobent noms, coutumes, objets de la vie égyptienne de la XVIII ${ }^{\mathrm{e}}$ ou XIX ${ }^{\mathrm{e}}$ dynastie $(\sim 1300)^{1}$. Cette matrice engendrera non seulement d'autres textes, mais aussi d'autres communautés, porteuses des écrits anciens et nouveaux, de conseils et de commandements ${ }^{2}$.

Il faut éclaircir ce rapport du multiple au singulier, qui est aussi celui de l'exclusion à l'élection, car c'est lui qui rend possible les métamorphoses successives. Tout d'abord, Joseph représente la figure de la victime émissaire, c'est-à-dire de la victime persécutée et violemment expulsée (Girard, 1999: 178). Le récit joséphique est un des nombreux récits bibliques qui dénoncent les phénomènes victimaires du tous contre un. Ce trait commun à beaucoup de textes bibliques semble annuler sa singularité; en outre, comment la victime singulière en vient-elle à porter multiplicité et universalité? Le récit joséphique aboutit non à la mise à mort de la victime, mais à un pardon par une série de substitutions 3; Joseph est une victime singulière en ce qu'il ne reconduit pas les processus victimaires mais y échappe par le pardon et par sa compréhension de ces processus. Cette singularité définit l'élection; l'exclu se métamorphose en élu: l'ambiguïté du récit, les catastrophes subies par ceux qui se reconnaissent en Joseph ne sont jamais tragiques, car l'histoire initiale finit sans "happy end", mais en évitant le fratricide, elle peut ainsi continuer.

6 Le cycle commence par la série des substitutions comme moindre mal: au fratricide fut substitué par Ruben le puits asséché, au puits la vente par Juda, aux vingt pièces des Ismaélites la tunique ensanglantée, au sang de Joseph le sang d'un chevreau, aux Ismaélites les Madianites, aux Madianites Potiphar. En Egypte, les substitutions continuent à amoindrir le mal: à l'amant espéré par la femme de Potiphar se substitue le vêtement déchiré dans la fuite de Joseph, à la mort se substitue la prison, à l'esclavage l'intendance, aux rêves angoissants des vaches et des épis les solutions de Joseph, à Pharaon se substitue Joseph, à la mort et à l'esclavage la vente des terres par les Egyptiens. La substitution de Juda clôt la série finale pour une fraternité à laquelle rien ne peut plus être substitué: à Benjamin est substitué Simon, au blé l'argent, à la coupe Benjamin, à Benjamin Juda (après la mauvaise tentative de substitution de Ruben). L'élection se substitue à l'exclusion.

7 Le jeu des substitutions, le sacrifice fait par Juda, évitent la permutation de la fraternité en hostilité; la fraternité est définitivement ancrée et on est sorti du cercle infernal des expulsions victimaires et des fratricides. La Maison d'Israël peut devenir peuple d'Israël, am segoula, peuple élu ou plutôt singulier. C'est la fraternité et non le fratricide qui permet aux douze tribus de se métamorphoser en peuple. Dans Juda, origine du nom juif, se joue la formation du peuple et son caractère éthique. Juda incarne la royauté 
messianique fraternelle (il a vendu Joseph et pris la place de Benjamin: deux figures messianiques et royales). En appelant Judas le traitre qui vend Jésus, on voit comment le christianisme a mis en place l'accusation des Juifs qui tiennent leur nom de Juda: la vente qui est une substitution salvatrice devient un acte ignoble et condamnable (Serres, 1980: 219). En revanche, les évangélistes réfèrent nettement Jésus, messie, aux deux branches messianiques juives, fils de Joseph, de la maison de David, c'est-à-dire de Juda.

Le récit joséphique est à la fois le texte qui donne la formation de la nationalité juive et le texte qui donne la formule du Juif parmi les Nations, tantôt persécuté, tantôt élevé aux plus hautes responsabilités, tantôt considéré comme assimilable, international, sans appartenance, cosmopolite, tantôt vu comme l'étranger irréductible. Joseph est Egyptien, Tsaphnat-Panéah, mais aussi Israël, car, vendu comme esclave, il quitte le lignage de son père, Israël, mais y voit réintégrés ses fils, Ménassé et Ephraïm, adoptés comme fils par leur grand-père (Genèse 48 5): un fils perdu devient deux tribus, dont une est royale (Baermann Steiner, 1954: 73-75). Tantôt en trop, tantôt en plus, Joseph, qui signifie en hébreu l'ajout mais aussi l'effacement, est à mettre en rapport avec Moïse le lévite (qui symbolise le retrait, la constitution sabbatique des lois). Ainsi, contrairement à ce qu'affirmait Jean-Paul Sartre dans ses Réflexions sur la question juive ${ }^{4}$, l'être d'Israël n'est pas essentiellement une identité forgée dans la constance des persécutions extérieures, d'abord compilées dans la Bible, même s'il entretient un rapport éminent à la question de la persécution.

9 Le questionnement moral qui en découle est un trait typique de la Bible; Nietzsche a bien relevé, pour la critiquer, ce qu'il appelle la "morale des faibles"; dès le début du Pentateuque, la réécriture des mythes sumériens dans la Genèse se marque par une métamorphose morale des ressorts du récit (la cause du déluge qui est, dans les premiers mythes, le boucan fait par les hommes, devient bibliquement leur perversité). L'être d'Israël, à travers son texte fondateur, est intimement lié à une exigence morale qui remet toujours en question la moralité des actions, comme en attestent les différents livres des prophètes; cela ne signifie pas une identification d'Israël et de la moralité, ni même une définition d'une essence éthique du judaïsme, mais une problématisation de son existence même en fonction de prescriptions morales, où le refus des accusations mensongères tient une place déterminante: les "faibles" dans cette morale sont les victimes innocentes d'accusations mensongères qui se polarisent sur une différence, une "faiblesse".

Joseph serait-il alors la revanche triomphale de l'Elu, du peuple élu sur les Nations? Dans le récit s'affrontent en fait deux types de fondation du peuple: une fraternité qui fonde l'appartenance fraternelle sur l'exclusion, voire le fratricide; une autre fraternité qui parvient à surmonter l'exclusion dans le pardon. Les fils d'Israël sont capables, comme toute nation, de la première fraternité exclusive, accusatoire, mais ils ont commencé par éviter le fratricide. Ils ne deviennent peuple d'Israël que dans une fraternité non exclusive, non accusatrice, bienveillante pour les Nations, même si elles ne sont pas fondées sur cette seconde fraternité qui serait, semble-t-il, la fraternité messianique.

11 L'être d'Israël se rapporte donc à la connaissance des logiques de persécution dont personne n'est exempt, pas même Israël; cette connaissance donne malgré tout une chance d'y échapper, mais elle ne s'acquiert qu'au bout d'une histoire, d'un récit initiatique, d'une répétition, de métamorphoses. La narration y est essentielle car, en théorie, les logiques de l'agir sont faciles à repérer, mais en pratique, face à l'urgence de l'action, à la diversité des situations, il est plus difficile de faire descendre la généralité 
des connaissances morales dans la fine pointe d'une réalité vivante et changeante, et plus facile aussi de réclamer pour soi l'exception à la règle qui ne saurait s'appliquer dans toute sa rigueur à tous les cas. Il y faut un conseil. Paradoxalement, c'est la persécution, ou la narration de la persécution, qui peut être facteur de clôture politique pour Israël, qui ouvre en même temps un messianisme universel, par le rejet des logiques de persécution dans les rebondissements d'une histoire comme celle de Joseph. Le récit de Joseph, modèle pour les Juifs de la diaspora, redouble cette universalité en se traduisant dans toutes les langues: son caractère messianique engendre constamment les métamorphoses linguistiques qui permettent son appropriation.

Pour Joseph, l'épreuve se répète plus d'une fois, jusqu'à ce qu'il en ait compris le sens; c'est alors que lui-même fait subir des épreuves à ses frères. Joseph n'accède pas à sa place lorsqu'on a fini de le persécuter, mais lorsqu'il a fini par comprendre les logiques de l'exclusion: l'échanson ingrat qui le fait venir auprès de Pharaon le fait pour son propre profit, mais Joseph, lui, ne cherche pas à tirer profit, à se présenter comme détenteur d'un savoir sur le bon et le mauvais, comme un Juste rigoureux, seul détenteur du Bien; il a appris de tous les épisodes précédents où il le faisait encore. Dans la perspective biblique, Joseph pouvait ainsi servir de modèle pour tout Juif vivant au sein des Nations.

Un paradoxe mérite alors d'être souligné: le récit dans lequel se met en place le schéma de composition complexe et singulier du peuple juif a servi plus qu'aucun autre à justifier la transmission de l'élection divine à d'autres peuples, à des communautés universelles. Dans ce paradoxe se fait jour le principe joséphique, complémentaire du principe lévitique, mosaïque. Joseph est présenté par la tradition juive comme le Juste qui anticipe les prescriptions mosaïques, maintenant la morale des patriarches dans l'extériorité idolâtre, appliquant (ou infligeant) des mesures lévitiques à l'Egypte. Joseph, figure du Juif tourné vers les Nations, est pour Israël celui qui détient l'aînesse spirituelle (1 Chroniques 5 1-2) et non politique, qui est celle de Juda, alors que son rôle pour les Nations est justement politique, qu'il soit conseiller ou vice-roi; rôle ambigu car le salut de l'Egypte passe alors par la dépossession des Egyptiens au profit de Pharaon et aboutit à l'esclavage des Hébreux.

14 Cette composition du peuple juif qui se met en place à la fin de la Genèse est complexe car elle suit tout un jeu d'ajouts et de retraits; ce jeu ne se résume pas au bariolage des douze tribus: il a un sens messianique et initie les rapports complexes et dangereux entre les autorités spirituelles et politiques. Joseph, qui a pris la place de sa demi- sœur Dina, qui est remplacé par ses deux fils dans le décompte des tribus, représente les dix tribus qui se perdront avec le royaume d'Israël; Benjamin verra le Temple construit sur son territoire et donnera le premier roi Saül; à Juda revient la royauté et le peuple juif lui doit son nom, Lévi enfin ne reçoit de part qu'à travers la dîme, dispersé parmi les tribus, dévolu à l'enseignement et au service divin.

La métamorphose de l'exclu en élu, sauveur des Hébreux et des Nations, fait de Joseph une figure messianique vouée à dépasser les limites du peuple juif: pour les chrétiens, Joseph est une préfiguration du Christ, et, pour les musulmans, avec la sourate $12 \mathrm{du}$ Coran, il préfigure Muhammad. Or, la figure messianique est incluse dans le récit biblique en Joseph, mais aussi en Juda; le récit de Joseph est coupé par l'histoire de Juda et de sa bru Tamar, ancêtres incestueux de David et fondateurs de cette lignée messianique. On comprend que les commentateurs chrétiens et les rédacteurs coraniques insistent sur la préfiguration joséphique plutôt que sur la préfiguration judaïque; selon ces deux 
traditions (ou traductions), la judéité est ce à quoi christianisme et islam doivent se substituer.

Un autre paradoxe découle du précédent: l'interprétation typologique chrétienne ou coranique marque une rupture dans l'exégèse du texte biblique et bouleverse l'économie des principes joséphique et lévitique. La rupture est relative dans le texte chrétien, car la Bible juive est conservée et l'histoire de Joseph fait partie des textes chrétiens; la vérité $\mathrm{du}$ texte joséphique se trouve cependant dans les Evangiles. Le texte musulman marque lui une rupture quasi-définitive puisque le Coran récrit l'histoire de Joseph; malgré l'usage du midrach, les textes juifs sont considérés comme des falsifications d'un texte divin rétabli par le Coran.

Dans les deux interprétations, c'est l'être politique juif qui disparaît. Dans l'exégèse chrétienne, l'aspect lévitique qui se rattache aux lois de Moïse est minoré, voire absent; ce qui est retenu, c'est l'attribution de l'aînesse aux cadets; l'esprit joséphique signe la perte de l'aînesse juive particulariste, lévitique, au profit du peuple chrétien universel. Dans l'interprétation coranique, c'est tout ce qui se rapporte au peuple juif qui est évacué pour se concentrer sur les embûches rencontrées par les prophètes (Prémare, 1989); la particularité juive était le "mensonge" et donc l'obstacle à la saisie universelle du message divin.

La tradition juive, quant à elle, ne marquant pas de rupture, qu'il s'agisse du midrach ou de la philosophie juive, ne clôt ni l'interprétation, ni la traduction. Certes, la vie de Joseph préfigure les dix commandements de Moïse, mais cette concordance qui est établie par le texte biblique lui-même n'empêche aucunement le développement de l'exégèse; la préfiguration des lois de Moïse n'est qu'une interprétation parmi d'autres, qui peuvent insister sur le fait que les lois du Sinaï ne sont pas encore données; une nouvelle interprétation est toujours possible. Les commentaires midrachiques et l'étude de l'organisation joséphique et lévitique du politique juif permettent à rebours de relire les Evangiles en changeant de perspective. Si la mort et la résurrection de celui qui est censé être le Messie ne focalisent plus l'attention, une continuité apparaît entre les enseignements de Jésus et ceux des rabbins de son temps.

L'opposition laisse place à un même souci des victimes, à une dénonciation de l'accusation mensongère et des processus d'exclusion. Au lieu qu'un récit préfigure l'autre, les récits se commentent les uns les autres, soulignant tel ou tel mécanisme victimaire, tel ou tel stéréotype de persécution ou d'accusation. Le texte de la rationalité s'en trouve considérablement étendu. Le Satan, l'Antéchrist, les textes de l'Apocalypse perdent ainsi leur aura mythologique; derrière les personnages, on trouve des concepts, des comportements mimétiques déterminés. Une fois établi que le texte joséphique désamorce accusations mensongères et processus victimaires, son caractère fondateur apparaît plus clairement.

Il permet de lier ensemble des notions généralement envisagées séparément: conseil, commandement et obéissance, clémence, pardon et fraternité, tolérance religieuse, cosmopolitisme et légitimation de l'autorité. Il devient alors plus aisé de discerner les auteurs qui se sont contentés d'enclore le sens de ce récit dans un autre récit, et ceux qui, répondant au commandement ou au conseil du texte joséphique, se saisissent de son autorité, en tirent un enseignement pour leur temps, en perçoivent la portée politique. Les historiens de la pensée et de la philosophie ont bien relevé les références au législateur Moïse chez nombre d'auteurs, penseurs et acteurs politiques (ce qu'on appelle le "lévitisme"), mais bien peu ont porté leur attention à la complémentarité des figures de 
Joseph et de Moïse chez plusieurs d'entre eux (ce qu'on peut appeler le "lévitisme joséphique").

21 Joseph assume ainsi une riche postérité théologico-politique, littéraire et herméneutique; certains auteurs vont jusqu'à s'identifier à lui, comme Flavius Josèphe, Voltaire ou Freud; une liste de tous les acteurs ou auteurs qui se rapportent à Joseph ou au récit joséphique serait non seulement fastidieuse, mais aussi incomplète ${ }^{5}$. Pour Philon d'Alexandrie, il incarne la politique et la citoyenneté juive; pour Calvin, il est le type de l'élu protestant; pour Grotius, il figure le républicanisme hollandais. Le récit joséphique inspire des écrivains comme Voltaire qui en fait le modèle premier du conte, mais aussi Goethe, Chateaubriand, Dumas, Péguy. Ce statut de modèle narratif permet de comprendre pourquoi ce texte est une source inépuisable de métamorphoses linguistiques. Thomas Mann, avec Joseph et ses frères, métamorphose la Genèse en roman pour former "l'humanisme de l'avenir" et lutter contre le fascisme. Franz Baermann Steiner, Ernst Bloch, Emmanuel Lévinas et René Girard repensent quant à eux le mythe biblique en termes de connaissance anthropologique libératrice.

Philon d'Alexandrie, contemporain de Jésus et de Caligula, est le penseur qui écrivit le seul livre de philosophie politique entièrement consacré à Joseph, ancêtre du préfet d'Egypte impérial': le De Iosepho. Philon, le platonicien, un des chefs de la communauté juive alexandrine, peut être considéré comme le fondateur de la philosophie juive, bien que, paradoxalement, sa postérité fût surtout chrétienne. Sa philosophie illustre bien en ce sens la singularité multiple des figures de Joseph. Celui-ci représente la figure éminemment ambiguë de l'homme politique, i. e. la diversité des systèmes politiques, la division politique humaine; le politique est alors un ajout néfaste à la perfection morale des trois patriarches et à la législation universelle de Moïse (Philon, 1964: 54-57).

La politique est le lieu de l'intermédiaire, de l'ambivalent où l'élection cache toujours une exclusion, mais cette position intermédiaire est aussi celle qui permet le conseil. Or, pour instituer une société juste, Philon, pourtant platonicien, ne se réfère pas à la République de Platon, mais à la société sabbatique, la République idéale de Moïse, qui évite les fondations meurtrières, exclusives, victimaires, totalitaires. Ainsi, dans le monde de l'adversité, les Lois de Moïse, conçues comme Lois de la Cité universelle, ont besoin de la politique joséphique pour conseiller, instituer, transporter à travers le temps la société sabbatique qui tente d'éviter les injustices. Il faut une politique joséphique qui résiste aux persécutions, quitte à prendre part à la politique des Nations, avec toute la prudence requise. Philon, philosophe et homme politique prudent, définit pour longtemps une dimension structurante de l'existence, de l'identité juives: "la citoyenneté juive universelle" (Philon, 1972: 204-205 (\$194)). Etre juif, c'est appartenir politiquement ou par ses origines à une autre nation non-juive, et spirituellement à la société sabbatique mosaïque; le paradoxe est ici que cette appartenance spirituelle a aussi une dimension politique, qui plus est universelle. Ce n'est pas l'appartenance juive qui est particulariste, c'est l'appartenance à telle ou telle nation. Aussi, les questionnements philoniens, à deux mille ans de distance, conservent leur acuité, et sont même actuels. La pensée philonienne, contre toute attente, nous place au cœur des problèmes de l'unité et de la division politiques et spirituelles, car, avec Joseph, le politique est pensé à partir du statut d'étranger résident. La politique joséphique, intermédiaire entre une intelligence politique et une intelligence prophétique, représente alors le Logos médiateur, la vie du sage obligé de résider en ce monde en étranger, obligé de participer à la vie politique 
pour l'élever, pour l'éclairer par l'intellect, pour interpréter les rêves éveillés des hommes engourdis dans le monde sensible (Philon, 1964: 93).

Pour Philon, ces politiques, qui ménagent une place à l'étranger, à la veuve, à l'orphelin, en somme au prochain, qui conseillent l'obéissance au lieu de l'imposer, appellent un régime démocratique et rejettent despotisme et démagogie. Le même intellect produit l'interprétation allégorique du texte biblique qui suppose l'univocité de son sens, qui est censée donner à son tour une unité politique face à la division politique et spirituelle des non- juifs. C'est cette lecture allégorique de la Bible qui fit la postérité de Philon dans la patristique chrétienne, délaissant son enseignement politique ou le réduisant à une moralité et une obéissance à Dieu des bons conseillers et gouvernants. Si on souligne le fait que Philon vivait au début de l'ère chrétienne, il est sans doute à regretter que la postérité de Philon fût théologique et allégorique, et non politique.

Après Philon et l'effondrement de l'établissement politique juif antique, l'histoire de Joseph retiendra surtout l'attention des théologiens: préfiguration, allégorie messianiques, juives, chrétiennes ou musulmanes, le conseil joséphique ne concerne plus qu'une élection, une "politique de l'immortalité". Si Joseph est une figure de la survie pour les Juifs de Philon et Flavius Josèphe à Isaac Abravanel, il faut attendre les bouleversements de la Renaissance pour que la figure joséphique retrouve son poids politique. Calvin, continuant pourtant à voir en Joseph la préfiguration, "la vive effigie" du Christ (Calvin, 1978: 513), retrouve l'analyse de la persécution qu'il rapporte à la situation morale et politique des protestants; or Calvin n'est pas qu'un théologien, il eut un rôle politique effectif.

Comme avec Philon, c'est la législation mosaïque qui sert de modèle d'institution, mais le modèle joséphique permet de concevoir l'attitude politique requise pour celui qui, réformant la société en vue d'une finalité plus haute, subit des persécutions (Calvin, 1957: livre IV, chapitre XX). Le commentaire de la Genèse, où Calvin porte une attention soutenue au récit joséphique, rassemble certes tous les grands thèmes de la théologie calviniste dont la fameuse double prédestination, mais il met aussi au jour la position atypique du législateur genevois dans son rapport au judaïsme; pas d'antisémitisme chez Calvin et peu d'antijudaïsme (étonnante singularité). La politique de Calvin promeut un régime mixte, un régime de conseil, et un modèle de société fondé sur le contrat, en suivant le modèle de l'Alliance mosaïque: là aussi Moïse et Joseph sont réunis.

Cette réévaluation inédite et isolée du judaïsme, alliée à une méfiance à l'égard du mysticisme, s'accompagne pourtant d'un fanatisme peu commun, qui tranche violemment sur la tradition protestante, dont il est pourtant l'un des maitres. Tout en analysant les processus victimaires dans son commentaire de l'histoire de Joseph ${ }^{7}$ (Calvin, 1978: 493, 516 et 527-528), Calvin était capable de persécuter de nombreux hommes au nom de ce qu'il croyait être le bien et le vrai (Michel Servet, Sébastien Castellion et tous les Genevois qui rechignaient devant l'inquisition des institutions calvinistes). Le lévitisme joséphique de Calvin, où l'unité spirituelle doit produire une unité politique, n'offre donc aucune garantie contre les tentations théocratiques ou totalitaires ${ }^{8}$.

Dans ce contexte protestant, le calviniste hollandais Grotius, après avoir composé son célèbre ouvrage juridique De iure belli ac pacis (1625) en France et travaillé (un peu) pour Richelieu, écrit une pièce qui sera un grand succès théâtral à Amsterdam pendant plusieurs décennies: Sophompaneas (1635). Exilé en France après des déboires politiques, il est définitivement considéré comme persona non grata en Hollande. Patriote, il hésite à se mettre au service d'une autre nation, ce qu'il fera finalement en devenant ambassadeur 
de Suède en France, sans doute encouragé par sa méditation de l'épisode biblique - un calviniste lettré ne pouvait ignorer les commentaires favorables de Calvin sur Joseph "droit interprète de la providence de Dieu" (Calvin, 1978: 590) et homme politique élu de Dieu. Mettant en scène les retrouvailles de Joseph et de ses frères, cette pièce permet à cet humaniste, juriste, homme politique déçu et exilé, mais toujours attaché à sa patrie, d'exprimer ses idées politiques en promouvant le républicanisme hollandais et en critiquant la manière de gouverner d'un Richelieu qu'il connaissait bien. En dépeignant l'Egypte sous les traits des institutions de la république hollandaise et de son réseau de cités, Grotius, dans sa préface, oppose Joseph, de son nom égyptien (s'inspirant de sa translittération dans le grec de la Septante), Sophompaneas, aux hommes politiques rusés, qui négligent la loi naturelle; il est assez vraisemblable qu'il visait son ancien employeur, Richelieu, dont il ne partageait pas les conceptions en matière de raison d'Etat et de "tolérance" religieuse.

Le pardon de Joseph et l'installation des frères en Egypte traitent d'ailleurs de cette question, lorsque Pharaon promet que "les Hébreux seront toujours libres de pratiquer leurs rites." (Grotius, 1992: 1. 1192-95). La pièce de Grotius métamorphose donc l'Egypte en république hollandaise et Joseph en figure rivale du premier ministre tout-puissant de Louis XIII, figure rivale que Grotius aurait rêvé d'incarner, mais qu'il dut se contenter de représenter. Tout au moins sa philosophie politique reçut un vaste écho par les très nombreuses représentations qui drainèrent une foule nombreuse sur près de quatre décennies au Schouwburg d'Amsterdam (Lang, 2009: 203); Grotius ne devait pas être le seul Hollandais à se reconnaitre dans l'histoire de Joseph ${ }^{9}$, mais il sut traduire les aspirations de liberté et de tolérance de ses contemporains dans la grande capitale européenne du XVIIe siècle, alors même qu'il ne pouvait plus y revenir.

Un fait frappant mérite de revenir à Richelieu. Le cardinal requit les conseils de Grotius lors de son exil parisien pour des questions de droit international, de commerce maritime, suivant le juriste dans l'idée que le commerce international et la mer doivent être libres, contre les prétentions habsbourgeoises (Lang, 2009: 192-193). La raison d'Etat française coïncidait sur ce point avec les intérêts hollandais, et ne dédaignait ni les conseils d'un jusnaturaliste calviniste hollandais ${ }^{10}$, ni la justification de ses exigences par la loi naturelle. Richelieu fut donc en rapport avec un conseiller pour qui Joseph avait un rôle important dans sa pensée et sans doute même dans sa vie. Ce rapport serait sans intérêt si le conseiller personnel, ami et soutien de Richelieu, n'était autre que le Père Joseph de Paris, la fameuse "éminence grise" ${ }^{11}$. Deux conseillers de Richelieu renvoient ainsi à la figure joséphique, mais avec des buts politiques et spirituels opposés. Avec Richelieu qui œuvrait à la centralisation de l'Etat dans la monarchie absolue française et combattait les protestants français, le Père Joseph, capucin mystique, croyait dans l'élection de la France à la tête de la catholicité. Cette élection de la nation française se rapportait de manière caractéristique à la figure de Joseph, depuis le Père Joseph et Bossuet, jusqu'à Charles Péguy, qui en fut peut-être le dernier représentant ${ }^{12}$.

31 Ainsi, deux éminentes figures catholiques joséphiques, deux conseillers ont joué un rôle politique de premier plan dans le développement de la monarchie absolue en France au XVIIe siècle, concevant la France comme la Nation élue. Qu'il s'agisse du Père Joseph (qui soutient Richelieu dans la prise de La Rochelle, conduisant à un affaiblissement définitif des protestants français) ou de Bossuet (qui convertit les protestants et conseille au roi la révocation de l'Edit de Nantes), une mystique unitive, un messianisme sous-tendent et arment une politique de puissance, la domination de la France sur l'Europe. Du Père 
Joseph de Paris et de Bossuet, la métamorphose de la figure joséphique la plus intéressante, et la plus inquiétante, est celle du Père Joseph.

Ce noble érudit, moine capucin mystique, devient le conseiller et l'ami de Richelieu; il est ainsi connu comme l'éminence grise (le Père Joseph de Paris était capucin: son habit était donc une robe grise; outre le fait qu'il était comme l'ombre du cardinal Richelieu, il devait lui-même être créé cardinal et donc "éminence"; mais la mort l'en empêcha): il mit en place un réseau d'espionnage européen grâce à son ordre monastique; il développa une politique de puissance et d'alliances où la fin justifie les moyens; l'éminence grise n'entendait pas laisser l'Espagne se charger de la tâche messianique. François Le Clerc du Tremblay prit le nom de Joseph en entrant dans les ordres; même si son saint patron est le père de Jésus, ce baron lettré, qui avait quelques rudiments d'hébreu, connaissait le sens du nom qu'il avait choisi et ne pouvait manquer de rapprocher son activité de conseiller et d'homme d'Etat de l'activité du conseiller biblique élu de Dieu, interprète privilégié de la providence divine.

Dans le même contexte de guerres, de troubles religieux et civils, un philosophe conseiller anglais, réfléchissant comme Grotius sur le droit naturel, en tire des conclusions absolutistes plus proches des conceptions de Richelieu que de celles du républicain hollandais. La philosophie contractualiste de Hobbes utilise parcimonieusement le récit et la figure joséphiques (Hobbes, 1971: 264, 421, 445, 557, 566). Cela ne signifie pourtant pas que la persona joséphique soit d'une importance secondaire. La relative discrétion de la figure joséphique tient à l'usage que Hobbes fait de l'Ecriture. Le philosophe entend confirmer par l'Ecriture les principes qu'il dégage rationnellement, sans justifier une autorisation du pouvoir souverain par un pouvoir spirituel quelconque. La théologie séculière hobbesienne use des personnages bibliques comme Joseph pour personnifier des principes (Hobbes, 1971: chapitres XXIV et XXV), comme le Léviathan personnifie l'Etat. Les lois de nature, la différence entre conseil et commandement peuvent être personnifiées par le récit joséphique; c'est l'autorité du philosophe que de décider des chapitres, des définitions où les personnifications, les citations bibliques apparaîtront pour augmenter l'autorité du souverain.

Le rapport à une transcendance ne saurait autoriser aucun pouvoir, sinon le pouvoir souverain sous forme de conseil, en veillant bien à maintenir la différence entre conseil et commandement. Evidemment, ce n'est pas la Bible qui donne à Hobbes ses principes politiques, mais il n'en reste pas moins que les récits bibliques, dont celui de Joseph, offrent une traduction adéquate à la pensée de Hobbes qui s'en nourrit. Certaines idées sont bien communes à Hobbes et à la pensée biblique: les hommes sont essentiellement égaux; les hommes rivalisent et s'entredétruisent; une alliance avec une autorité est conjointe à une alliance des hommes entre eux; le conseil d'obéir à une autorité suppose que l'obéissance ne soit jamais aveugle. L'œuvre de Hobbes constitue donc un modèle pour l'utilisation du théologique en politique, là où ce qui importe est l'unité politique.

Il est significatif que des conseillers du XVIIe siècle, théologiens ou philosophes, se soient référés à la figure de Joseph, avec des intentions différentes, voire opposées; ils manifestent tous une métamorphose de la figure de Joseph dont le récit devient le support d'une théologie séculière, où le politique prend le pas sur le théologique. Le fait qu'une figure biblique initie une réflexion politique où le théologique est utilisé à des fins non théologiques nous montre bien la naissance de la politique moderne, la métamorphose des Etats-nations européens au XVIIe siècle, et nous place au cœur du débat sur la sécularisation. 

21), fixa l'attention de plusieurs théologiens et philosophes: l'épisode du Lévite d'Ephraïm, si effrayant, ne favorisa pas l'intérêt pour le lévitisme joséphique et les conceptions politiques juives. Voltaire, dans son Dictionnaire philosophique ${ }^{13}$, n'y voit qu'une preuve de plus de l'ineptie politique des Juifs et de leur cruauté. Bossuet, avant lui, y voyait une preuve supplémentaire qu'un peuple ne saurait se passer de roi: "La seule autorité du gouvernement peut mettre un frein aux passions, et à la violence [...]. Quand chacun fait ce qu'il veut et n'a pour règle que ses désirs, tout va en confusion. Un lévite viole ce qu'il y a de plus saint dans la loy de Dieu. La cause qu'en donne l'écriture: "C'est qu'en ce temps-là il n'y avoit point de roy en Israël, et que chacun faisoit ce qu'il trouvoit à propos."“ (Bossuet, 1709: 22 (Livre I article III, proposition II)). épisode une image des premiers temps de l'humanité, "paradoxaux, puisque bonté et cruauté s'y enchevêtrent", là où "la violence à la fois détruit et fonde le corps social" (Rousseau, 2010: 17). Dans son introduction à l'ouvrage de Rousseau, Sébastien Labrusse résume "l'anthropologie politique" du Lévite d'Ephraïm: "la violence qui ravage les sociétés se manifeste par une dévastation du langage, et cette dévastation prend la forme de l'éclatement des corps vivants" (Rousseau, 2010: 17). Cet opuscule méconnu de Rousseau est bien une synthèse sous forme de narration de sa pensée politique et anthropologique, mais il ne constitue pas une approche du lévitisme joséphique, puisque l'absence de souveraineté dans le récit des Juges n'est comprise qu'en terme de chaos et d'absence de droit. Il semblerait alors que la réflexion politique à partir de la figure joséphique fût vouée à se tarir à partir du moment où la "sécularisation" des sociétés occidentales progresse.

On pourrait ainsi considérer que la figure joséphique se résume à une question théologico-politique qui s'éteindrait à l'aube de la modernité occidentale; il n'en est rien.

Outre le regain d'intérêt pour le récit et ses potentialités romanesques qui se développa dans le sillage de la campagne d'Egypte napoléonienne et l'orientalisme lié au colonialisme, la figure joséphique est plus que jamais présente au XXe siècle et chez les plus grands. Freud dans L'Interprétation des rêves s'identifie à Joseph, l'interprète des rêves; dans ses rêves, des Joseph apparaissent qui sont des masques transparents pour son propre moi. La psychanalyse se révèle ainsi joséphique et se rapproche de la tradition juive d'interprétation; Joseph survit en interprétant, car ses premières interprétations de rêves ont manqué le tuer; Joseph fait survivre un des deux prisonniers, puis l'Egypte et les Hébreux. L'interprétation juive n'est pas une quête d'immortalité, elle a toujours un sens vital où les principes de l'interprétation, qui sont en même temps éthiques, sont souvent tournés vers des explications singulières, des événements, des questions pratiques pour ici et maintenant ${ }^{14}$. Les diverses métamorphoses linguistiques du récit joséphique peuvent ainsi être comprises comme des réinterprétations qui libèrent un nouveau sens du récit, une nouvelle manière de le dire; parole singulière qui libère, et non parole tragique qui condamne.

40

Freud désire savoir quel désir est signifié par le rêve comme Pharaon et parvient à interpréter comme Joseph. Celui-ci, ainsi que le souligne Freud (Freud, 1980: 287), anticipe déjà la théorie freudienne, il connaît la causalité des désirs figurés dans le rêve, puisqu'il explique, avant Freud ${ }^{15}$, que "plusieurs rêves apparus au cours d'une même nuit" ont une même "signification" (Freud, 1980: 287). Freud est proche de Joseph car, 
comme il le dit lui même en expliquant le titre de son ouvrage (Freud, 1980: 90), il est plus proche du sens commun que des savants: le rêve a une signification, on peut la trouver.

41 Freud se rêve en Joseph, c'est-à-dire celui qui sait interpréter les rêves; il retrouve par là la position de Flavius Josèphe qui réclamait la même identification à Joseph lors de la chute du temple et de Jérusalem. Flavius Josèphe se rend à Vespasien, fait un rêve et lui prédit l'empire; ce dernier passe pour un traître auprès des Juifs, mais il est de fait la source historique presque unique pour le judaïsme du Ier siècle. On peut interpréter Joseph comme une condensation de l'Hébreu de la marge qui interprète les rêves pharaoniques et rejoint le centre du pouvoir (Sloterdijk, 2005: 29-32); Freud n'est-il pas un Hébreu de la marge (la Moravie) qui interprète les rêves dans la capitale impériale des Habsbourg et réussit une brillante ascension sociale? Une telle identification joséphique ne pouvait que conduire à une réévaluation totale de la figure juive de Moïse qui paraitt prendre le contrepied de la figure joséphique; surtout au moment où, victime du nazisme ${ }^{16}$, Freud est contraint à l'exil au Royaume-Uni. Le Joseph viennois doit lui aussi transporter et conserver la psychanalyse avec lui.

On sait que Moïse est l'objet d'un mythe, d'une réécriture ou plutôt d'un déplacement ("Entstellung") ${ }^{17}$ où la judéité de Freud est impliquée, que Freud craignait que la psychanalyse ne passât pour une "science juive". Il voulait déraciner l'idée que les Juifs avaient inventé le monothéisme et désamorcer ainsi la rivalité avec tous ceux qui se considéraient comme élus. Ce faisant, Freud entendait réaffirmer, à l'instar de Max Weber et comme Thomas Mann, l'idée que le judaïsme constituait une des sources de la pensée rationnelle et éthique, de la civilisation européenne (Le Rider, 2005: 59-66); Moïse l'Egyptien renouait avec l'égyptophilie du récit joséphique où l'Hébreu est ouvert aux Nations, les nourrit, les protège. Le déplacement de Moïse vers Akhenaton (souverain de la XVIIIe dynastie) permettait de conserver la portée éthique universelle de la psychanalyse tout en confirmant son apport à la pensée scientifique; ceci à l'heure même où les antisémites renvoyaient la psychanalyse à la judéité et les psychanalystes, pour beaucoup juifs, à leur prétendue "race". Freud semble vouloir conserver les principes de l'élection sans l'élection qui particularise le peuple juif et le voue à l'exclusion.

Le refoulé juif de la pensée freudienne fait retour en Joseph; le déplacement, la métamorphose opérés par Freud sur la figure de Moïse souligne le fait que la figure joséphique est présente en creux dans l'œuvre de Freud sans qu'il ait véritablement pris en charge cette figure. Aperçue, elle déclenche un désir spéculatif qui se tarit avec son examen; Freud semble avoir écarté de sa réflexion un récit qui aurait dû l'alimenter. Freud rédigeait son Moïse parallèlement au Joseph de Thomas Mann et se disait très stimulé par les travaux et le roman de ce dernier, mais il n'en est resté pour nous qu'une lettre où le destin joséphique de Napoléon Bonaparte est esquissé par le psychanalyste (Freud, 1966: 471- 473). C'est en fait avec Thomas Mann que Joseph acquiert la stature freudienne qu'il mérite en tentant de résorber le malaise dans la civilisation.

Thomas Mann est celui qui a récrit l'histoire de Joseph pour déraciner le mal de son temps, pour lever les accusations mensongères, dénoncer les persécutions dans une "Fête de la narration" (Mann, 1936: 47). Certes, le travail pyramidal de Mann, la tétralogie de Joseph et ses frères, est ambigu et semble vouloir remplacer la Bible mosaïque par une Bible joséphique (Pietra, 2011: 346-379), censée être plus universelle: le juif, l'élu, se fait joker, figure de substitution, qui réfracte en lui toutes les figures mythologiques de toutes les Nations, dont aucune n'est exclue ${ }^{18}$. Ce "mythe vécu" (Jung, Kerényi, 1968: 16-18) qui relève l'élément "typique-mythique" (Mann, 1960: 202-204) de toute vie humaine peut 
laisser perplexe; dans "un roman mythique comme le Joseph", "la fonction du mythe y était l'objet d'un renversement dont on ne l'eût pas cru capable. Cela ressemblait fort à ce qu'on observe dans les batailles lorsque les canons conquis à l'ennemi sont retournés contre lui" (Mann, 2006: 139).

Le joséphisme hermétique de Mann, le "jeu" avec le texte biblique (Mann, 2006: 135), pourraient rendre la leçon politique difficile à tirer; la politique de Thomas Mann n'est pas un commandement; elle est de l'ordre joséphique du conseil. Il faut ainsi retenir l'exigence spirituelle de Mann qui favorisait l'acceptation de la plus grande diversité spirituelle, le refus de toute exclusion, qui redonnait au judaïsme une place essentielle dans la conscience européenne et occidentale: "Le mythe, dans ce livre, était arraché aux mains du fascisme et humanisé jusque dans les derniers recoins du langage" (Mann, 2006: 139) ${ }^{19}$. Cette exigence spirituelle, qui était aussi ironie, transforma le jeune auteur réactionnaire en soutien de la démocratie sociale et de $\mathrm{F}$. D. Roosevelt, nommément identifié à Joseph, dans une union politique face à ceux qu'il appelait "les partisans de la bassesse" (Mann, 1970: 196). Avec Joseph et ses frères, il traçait la figure d'un christianisme universel, laïcisé, réfractant toutes les aspirations religieuses qui délivrent du mal, de l'exclusion de l'autre homme.

L'ethnologue et poète Franz Baermann Steiner définissait Joseph comme "le premier à perdre son pays natal [heimat]" (Baermann Steiner, 2000: 358-359); selon lui, la fin de la Genèse est "le plus ancien récit de la métamorphose d'un homme qui, à travers de cruels revers, par la volonté de son berger, subit des purifications" (Baermann Steiner, 2009: 172). Echapper au mal, aux persécutions empêche l'enracinement autochtone et semble toujours fournir les bases d'une nouvelle institution, d'un nouveau récit; on comprend aisément que la répétition universelle de cette expérience humaine à travers l'Histoire a pu produire les métamorphoses constantes d'un récit qui portait constitutivement ces potentialités en portant conseil. La méditation de ces métamorphoses, y compris de leurs ambiguités, n'a pas pour seul intérêt de former une catégorie théologico-politique (le lévitisme joséphique), elle conduit à renouveler nos conceptions de la fraternité humaine, en prenant conscience que la sagesse politique est de l'ordre du conseil, et de la narration. Ces conseils, ces narrations fournissent une connaissance universalisable sans exclusion.

\section{BIBLIOGRAPHIE}

Ouvrages critiques et théoriques:

ABEL, Olivier et SMYTH, Françoise (dir.) (1992). Le Livre de traverse. Paris: Cerf.

BAERMANN STEINER, Franz (May 1954). "Enslavement and the Early Hebrew Lineage System: An Explanation of Genesis 47: 29-31, 48: 1-16". In: Man, Royal Anthropological Institute of Great Britain and Ireland, Vol. 54, pp. 73-75.

BAERMANN STEINER, Franz (2009). Feststellungen und Versuche. Aufzeichnungen 1943-1952. Hrsg. v. U. van Loyen und E. Schüttpelz. Göttingen: Wallstein Verlag. 
BENJAMIN, Walter (1995). Rastelli raconte et autre récits suivi de Le narrateur. Paris: Seuil.

BILLON, DAHAN, LE BOULLUEC (déc. 2004). Le roman de Joseph (Genèse 37-50). In: Cahier Evangile, Paris: Cerf, supplément $\mathrm{n}^{\circ} 130$.

BLOCH, Ernst (1985). Briefe 1903-1975. Bd. 2. Frankfurt am Main: Surhkamp.

BLOCH, Ernst (1991). Le Principe Espérance. Tome III. Paris: Gallimard.

BOSSUET, Jacques-Bénigne (1709). Politique tirée des propres paroles de l'écriture sainte à Mgr le dauphin. Paris: Pierre Cot.

CALVIN, Jean (1978). Commentaires de Jean Calvin sur l'Ancien Testament, Tome premier, Le livre de la Genèse. Aix-en-Provence: Editions Kerygma.

CALVIN, Jean (1957). Institution de la religion chrestienne. Paris: Vrin.

FREUD, Sigmund (1966). Correspondance 1873-1939. Paris: Gallimard.

FREUD, Sigmund (1986). L'homme Moïse et la religion monothéiste. Paris: Gallimard, Folio/Essais.

FREUD, Sigmund (1980). L'interprétation des rêves. Paris: PUF.

GIRARD, René (1999). Je vois Satan tomber comme l'éclair. Paris: Grasset.

GIRARD, René (1982). Le bouc émissaire. Paris: Grasset. HOBBES, Thomas (1971). Léviathan. Paris: Sirey.

JANET, Pierre (1936). L'intelligence avant le langage. Paris: Flammarion.

JUNG, Carl Gustav, KERÉNYI, Karl (1968). Introduction à l'essence de la mythologie. Paris: Payot.

LANG Bernhard (2009). Joseph in Egypt. New Haven: Yale University Press.

LE RIDER, Jacques (1/2005). “Joseph et Moïse égyptiens: Sigmund Freud et Thomas Mann”. In: Savoirs et clinique, $n^{\circ} 6$.

LUKACS, Georg (1967). Thomas Mann. Paris: Maspero.

MANN, Thomas (2006). Etudes. Paris: Gallimard.

MANN, Thomas (1960). “Freud et l'avenir”. In: Noblesse de l'esprit. Paris: A. Michel.

MANN, Thomas (1970). Lettres de Thomas Mann. Paris: Gallimard.

NOCQUET, Dany (2002/1). “Genèse 37 et l'épreuve d'Israël, L'intention du cycle de Joseph”. In: Etudes théologiques et religieuses. $77^{\mathrm{e}}$ année.

Philon d'Alexandrie (1964). De Iosepho. Paris: Cerf.

Philon d'Alexandrie (1972). Legatio ad Caium. Paris: Cerf.

PIERRE, Benoist (2007). Le Père Joseph. Paris: Perrin.

PIETRA, Laurent (2011). Le conseil politique rapporté à la figure biblique de Joseph. Université Paris Ouest Nanterre La Défense. [consulté le 13/04/2013] <URL: http://bdr.u- paris10.fr/theses/ internet/2011PA100144.pdf>

PRÉMARE, Alfred-Louis de (1989). Joseph et Muhammad, le chapitre 12 du Coran. Aix-en- Provence: Publications de l'Université de Provence.

PURY, Albert de, RÖMER, Thomas (2002). Le Pentateuque en question. Genève: Labor et fides.

RICCEUR, Paul (1983-1985). Temps et récit. Tomes I, II, III. Paris: Le Seuil.

SARTRE, Jean-Paul (1954). Réflexions sur la question juive. Paris: Gallimard. 
SERRES, Michel (1980). Le parasite. Paris: Grasset.

SLOTERDIJK, Peter (2005). Derrida, un égyptien. Paris: Maren Sell Editeurs.

VERGOTE, Jozef (1959). Joseph en Egypte. Louvain: Presses Universitaires de Louvain.

VOLTAIRE (1964). Dictionnaire philosophique. Paris: Garnier-Flammarion.

VUILLET, Hélène (2007). Thomas Mann, Les métamorphoses d'Hermès. Paris: Presses de l'Université

Paris Sorbonne.

Ouvrages fictionnels:

BAERMANN Steiner, Franz (2000). Am stürzenden Pfad: Gesammelte Gedichte. Adler J. (Hg.).

Göttingen: Wallstein Verlag.

GROTIUS, Hugo (1992). Sophompaneas 1635, edited by Arthur Eyffinger (De Dichtwerken van Hugo Grotius). Assen: Van Gorcum.

HUXLEY, Aldous (2001). L'éminence grise. Paris: La table ronde.

LEFEBVRE, Gustave (1988). Romans et contes égyptiens de l'époque pharaonique. Paris: Maisonneuve.

MANN, Thomas (1974). Gesammelte Werke. Frankfurt am Main: S. Fischer.

MANN, Thomas (1936). Joseph et ses frères. Paris: Gallimard, Collection L'imaginaire.

PÉGUY, Charles (1957). CEuvres poétiques complètes. Paris: Gallimard, La Pléiade.

ROUSSEAU, Jean-Jacques (2010). Le Lévite d'Ephraïm. Chatou: La Transparence.

Textes biblique et coranique:

Bible de Jérusalem (1975). Paris: Desclée de Brouwer.

La Bible (1994). Traduction intégrale hébreu-français. Tel Aviv: Editions Sinaï.

Le Coran (1967). Paris: Gallimard.

\section{NOTES}

1. Les lecteurs soucieux d'approfondir la question des sources du récit joséphique ainsi que des exégèses rabbinique (midrachique) et historico-critique trouveront quelques références dans la bibliographie en fin d'article; ils peuvent aussi se reporter à un travail de thèse où ces questions sont abordées et discutées, et où une bibliographie plus conséquente est proposée (Pietra, 2011: 23-76).

2. Comme le souligne le psychologue Pierre Janet, "l'acte de la mémoire est une conservation et un transport d'un objet intellectuel important, il conserve et transporte le commandement. Le phénomène essentiel de la mémoire humaine, c'est l'acte du récit. Le récit est un langage et au fond un commandement, mais qui a des propriétés particulières, celle de permettre à des individus qui ont été absents au moment de certains événements de se comporter cependant comme s'ils avaient été présents, le récit transforme les absents en présents." (Janet, 1936: 163-164).

3. Dans son Dictionnaire philosophique, à l'article "Joseph" (Voltaire, 1964: 245-247), Voltaire le présente comme un "modèle", "l'un des plus précieux monuments de l'antiquité", qui "constitue un poème épique intéressant: exposition, nœud, reconnaissance, péripétie, et merveilleux: rien n'est plus marqué au coin du génie oriental." En outre, l'histoire de Joseph "qui pardonne" est plus "attendrissante" que celle d'Ulysse "qui se venge". 
4. "Le seul lien qui les unisse, c'est le mépris hostile où les tiennent les sociétés qui les entourent" (Sartre, 1991: 111) ou “c'est l'antisémite qui crée le Juif." (Sartre, 1991: 173).

5. La figure de Joseph ne cesse pas d'intéresser, jusqu'à aujourd'hui; on peut citer Peter Sloterdijk (2005) et son Derrida, un égyptien. Pour une liste assez conséquente, on pourra se référer à deux ouvrages: BILLON, DAHAN, LE BOULLUEC (déc. 2004). Le roman de Joseph (Genèse 37-50). In: Cahier Evangile, Paris: Cerf, supplément $n^{\circ} 130$, et LANG Bernhard (2009). Joseph in Egypt. New Haven: Yale University Press.

6. Fonction exercée par le propre neveu de Philon, citoyen romain et juif apostat, second de Titus: Tiberius Julius Alexander.

7. Selon Calvin, accuser quelqu'un pour ce qu'il est et non pour un mal effectivement commis, c'est se mettre "comme des brouillards devant les yeux", c'est faire "la guerre contre Dieu". Or, si des élus, comme les frères de Joseph, "ont été transportés d'un tel aveuglement, qu'adviendra-t-il aux réprouvés"? La même chose peut "nous" advenir "souvent tout comme à eux" (Calvin, 1978: 516).

8. Un peu avant Calvin, dans le contexte de la persécution et de l'expulsion des Juifs d'Espagne, le lévitisme joséphique d'Isaac Abravanel induit la même tentation théocratique, un idéal politique semblable et donne un sens messianique au récit joséphique, ce messianisme joséphique politique étant lui-même dépassé par le messianisme davidique non-politique. Il n'en reste pas moins que le commentaire de la Genèse permet à Abravanel, philosophe et rabbin, ancien conseiller et banquier des souverains espagnols, de résumer toute sa science du conseil politique.

9. On compte des dizaines de tableaux de Rembrandt prenant pour thème l'histoire de Joseph.

10. En fait, fondateur du "droit naturel (jus naturale)" moderne.

11. L'ouvrage d'Aldous Huxley, L'éminence grise (2001) n'est certes pas un travail universitaire (pour cela, voir celui de Benoist Pierre (2007)), mais il offre un abord plaisant à la biographie du conseiller mystique et machiavélique: il donne aussi une bonne illustration du topos "la réalité dépasse la fiction".

12. Charles Péguy publie dans les Cahiers de la Quinzaine en 1911 le Mystère des saints Innocents où il entremêle les histoires de Joseph, l'“avant", de Jésus, le "pendant" et de saint Louis, l'“après": "O théâtre d'Egypte, on y a joué trois fois./ Une fois avant. Une fois pendant. Une fois après."; on y retrouve donc le motif de la préfiguration messianique avec l'accent bien reconnaissable de la poétique de Péguy: "Un homme avait deux fils. Un homme avait douze fils. / Et ainsi devant toute sœur chrétienne/ S'avance une sœur juive qui est sa sœur aînée et qui l'annonce et qui va devant." (Péguy, 1957: 797).

13. Article "Juifs", section I. France : BnF-Gallica (texte numérisé), p. 446 [consulté le 28/04/2013] <URL: http://gallica.bnf.fr/ark:/12148/bpt6k375370/f546.image\#>.

14. Il semble que c'est ainsi que Freud caractérise lui aussi ce qu'il appelle "la religion de Moïse" (Freud, 1986: 225-227).

15. Dans le développement théorique, le personnage de Joseph semble perdre ici son caractère fictif; il est un interprète des rêves au même titre que l'historien juif Flavius Josèphe et que $C$. G. Jung cité dans l'alinéa suivant.

16. C'est Marie Bonaparte qui, grâce à ses relations, permettra la fuite de Freud en payant une rançon aux nazis.

17. "Dans bien des cas d'Entstellung de texte, nous pouvons donc nous attendre à trouver, caché ici ou là, l'élément réprimé et dénié, même s'il est modifié et arraché à son contexte." (Freud, 1986: 115)

18. Joseph est une "figure transparente" qui regroupe en lui "Adonis", "Tammouz" et "Hermès", de même que "son grand dialogue avec Pharaon mêle si bien toutes les mythologies du monde, hébraïque, babylonienne, égyptienne et grecque, qu'on aura peine à ne voir encore dans cette œuvre qu'un simple livre d'histoires bibliques et judaïques." (Mann, 2006: 147-148). 
19. Pour Ernst Bloch (1985: 703), "re-fonctionnaliser le mythe", "c'est modifier sa fonction sociale qui a été dévoyée, rétrécie, afin de le restaurer dans son universalité intrinsèque". Pour Mann (1974: 651 (Band XI)), "humaniser le mythe" consistait sans doute à passer du récit sacré, des "Saintes Ecritures", au récit profane, "bourgeois", au roman, mais le sens de cette formule est manifestement plus complexe et n'a pas la clarté d'un concept bien défini; ce sens ne peut être que conjecturé car il faut rassembler de nombreux éléments épars, laissés entre les lignes du roman et ses commentaires.

\section{RÉSUMÉS}

Les fictions et les différents ouvrages qui métamorphosent la figure de Joseph, loin de se limiter à un imaginaire littéraire, renouvellent le conseil joséphique, donnant à l'interprétation (des textes et des expériences précédents) le sens d'une survie, d'une échappée possible hors des maux d'une époque. Le présent article entend se placer dans cette longue "tradition" et poursuivre cette interprétation des textes joséphiques.

The different stories and works transforming the figure of Joseph, far from limiting itself to a mere literary imagination, renew the josephic counsel, where interpretation means survival and escaping from evil times. The present article intends to follow this long "tradition" and brings forward an advanced interpretation of the josephic texts.

\section{INDEX}

Mots-clés : Autorité, Conseil, Judaïsme, Narration, Philosophie, Politique

Keywords : Authority, Counsel, Judaism, Narrative, Philosophy, Politics

\section{AUTEUR}

\section{LAURENT PIETRA}

Sophiapol, Université Paris Ouest Nanterre

lapietra@numericable.fr 